OPEN ACCESS

Edited by:

Arran Turnbull,

University of Edinburgh,

United Kingdom

Reviewed by:

Lorenzo Gerratana,

University of Udine, Italy

Nicolò Matteo Luca Battisti,

Royal Marsden NHS Foundation Trust,

United Kingdom

*Correspondence:

Jin Yang

jingchi28@163.com

Specialty section: This article was submitted to

Women's Cancer,

a section of the journal

Frontiers in Oncology

Received: 20 December 2019

Accepted: 26 March 2020

Published: 30 April 2020

Citation:

Wang Y, Liu S, Zhang Y and Yang J (2020) Dysregulation of TLR2 Serves as a Prognostic Biomarker in Breast Cancer and Predicts Resistance to

Endocrine Therapy in the Luminal B

Subtype. Front. Oncol. 10:547.

doi: $10.3389 /$ fonc. 2020.00547

\section{Dysregulation of TLR2 Serves as a Prognostic Biomarker in Breast Cancer and Predicts Resistance to Endocrine Therapy in the Luminal B Subtype}

\author{
Yunmei Wang ${ }^{1,2}$, Shuguang Liu ${ }^{3}$, Yanjun Zhang ${ }^{2}$ and Jin Yang ${ }^{1 *}$ \\ ${ }^{1}$ Department of Medical Oncology, First Affiliated Hospital of Xi'an Jiaotong University, Xi'an, China, ${ }^{2}$ Shanxi Provincial \\ Cancer Hospital, Taiyuan, China, ${ }^{3}$ Department of Orthopedics, HongHui Hospital, Xi'an, China
}

Background: Breast cancer $(\mathrm{BCa})$ is a serious global health burden among females, and the development of resistance represents an important challenge to BCa treatment. Here, we examined the expression of toll-like receptor 2 (TLR2) in BCa patients and the prognostic value of TLR2 for predicting endocrine resistance.

Methods: The study included 150 BCa patients, of which 82 underwent endocrine therapy. TLR2 mRNA expression was measured by quantitative Real-Time PCR, and its prognostic value was determined by Kaplan-Meier survival analysis. Changes in the expression of TLR2 in BCa patients with endocrine resistance were assessed, and the value of TLR2 for predicting endocrine resistance was evaluated using the receiver operating characteristic curve analysis.

Results: TLR2 expression was higher in BCa tissue than in normal tissue and associated with tumor size, HER2 status, tumor subtype, and TNM stage. TLR2 upregulation was associated with poor prognosis in patients with $\mathrm{BCa}$, as well as endocrine resistance, and TLR2 upregulation was more prevalent among HER2-positive BCa cases. The predictive performance of TLR2 for endocrine resistance was higher in HER2-positive BCa than in other hormone receptor-positive BCa cases.

Conclusion: TLR2 upregulation is a promising biomarker for prognosis and predicting resistance to endocrine therapy. The relationship between TLR2 and HER2 indicates that TLR2 may be involved in endocrine resistance through the HER2 signaling pathway in $\mathrm{BCa}$.

Keywords: breast cancer, toll-like receptor 2, prognosis, endocrine therapy, resistance, luminal B subtype

\section{INTRODUCTION}

Breast cancer $(\mathrm{BCa})$ is the most frequently diagnosed malignancy and the leading cause of cancer-related mortality among women worldwide (1). There were 2,088,849 new BCa cases and 358,989 deaths from BCa in 2018, accounting for $24.2 \%$ of all cancer cases and $15.0 \%$ of total deaths among global malignancies in women (2). BCa can be divided into four major subtypes according to the expression status of the estrogen receptor (ER), progesterone receptor (PR), and the human 
epidermal growth factor receptor 2 (HER2), namely, luminal A, luminal B, HER2+, and triple-negative BCa (3). However, emerging data indicate an increase in the incidence of resistance to endocrine therapy, especially in luminal B BCa (4). Arpino et al. reported that the decreased sensitivity of luminal B BCa to endocrine therapy might be triggered by the crosstalk between ER and HER2 (5). Therefore, novel strategies to inhibit the activity of HER2 may increase the therapeutic efficacy of endocrine therapy in hormone receptor-positive $\mathrm{BCa}$ patients.

Toll-like receptors (TLRs) are a family of innate immune receptors that can recognize ligands derived from viruses, bacteria, parasites, and fungi (6). TLRs play important roles in the regulation of inflammatory responses aimed at eliminating pathogen infection and cancer debris (7). In addition, they play regulatory roles in cell proliferation and apoptosis in both normal and tumor cells $(8,9)$. Increasing evidence supports the double-edged sword effect of TLRs in cancer cells, because uncontrolled TLR signaling leads to a microenvironment that promotes tumor growth $(10,11)$. The TLR family is divided into two major subtypes according to cellular localization. TLR $1,2,5,6$, and 10 are extracellular TLRs, whereas TLR3, 7, 8, and 9 are localized in intracellular organelles (12). TLR2 and TLR4 are two major TLRs that have been widely investigated for their involvement in inflammatory responses and cancers (13). The expression and clinical significance of TLR4 have been reported in patients with $\mathrm{BCa}$ (14). TLR2 polymorphisms are associated with $\mathrm{BCa}$ risk (10), and TLR2 is involved in immunomodulation in $\mathrm{BCa}$ (15). TLR2 is upregulated in most tumors and closely associated with tumor metastasis, playing an important role as a proto-oncogene $(16,17)$. In colorectal cancer, TLR2 promotes tumor cell proliferation, migration, and invasion by activating the PI3K/AKT signaling pathway (18). However, the clinical significance of TLR2 in BCa remains unclear. Lu et al. showed that TLR2 agonists enhance the efficacy of HER2targeted monoclonal antibody therapy (19). Considering the important role of HER2 in endocrine therapy resistance in $\mathrm{BCa}$, we investigated the potential role of TLR2 in regulating the sensitivity to endocrine therapy in $\mathrm{BCa}$.

In the current study, changes in the expression of TLR2 in $\mathrm{BCa}$ patients and patients with endocrine therapy resistance were investigated, and the clinical significance of TLR2 as a prognostic factor for BCa was evaluated. In addition, we assessed the value of TLR2 for predicting endocrine therapy resistance in patients with luminal BCa.

\section{METHODS AND MATERIALS}

\section{Patients and Tissue Samples}

The study enrolled 150 patients who were histopathologically diagnosed with BCa in Shaanxi Provincial Cancer Hospital affiliated to the Medical School between 2011 and 2013. None of the patients received anti-tumor therapy before sampling. Of the $150 \mathrm{BCa}$ patients, 68 underwent surgical resection and 82 received endocrine therapy. $\mathrm{BCa}$ tissues and adjacent normal tissues were collected from the patients and frozen in liquid nitrogen for further use. The experimental procedures were approved by the Ethics Committee of Shaanxi Provincial Cancer
Hospital, and written informed consent was obtained from each participant.

\section{Clinical Data Collection}

The demographic and clinicopathological characteristics of the patients were recorded, including age, tumor size, ER, PR, and HER2 status; tumor subtypes; and TNM stage. ER, PR, and HER2 [FISH (Fluorescence in situ hybridization) was used to characterize HER2 expression in IHC (Immunohistochemistry) $2+$ cases] status was examined by immunohistochemistry, and BCa subtypes were categorized following the St. Gallen Expert Consensus as follows: luminal A (ER+ and/or PR+, HER2-, Ki$67<14 \%$ ), luminal B (ER+ and/or PR+, HER2-, Ki-67 $\geq 14 \%$; ER+ and/or PR+, HER2+), HER2+ (ER-, PR-, HER2+), and triple-negative (ER-, PR-, HER2-) (20). The TNM stage of BCa patients was determined according to the criteria published by the American Joint Committee on Cancer Classification (21). Overall survival (OS) was defined as the percentage of cases who had been alive after a number of months. The duration is from the beginning of filtering to death. In addition, survival information and the rates of endocrine therapy resistance [primary endocrine resistance was defined as recurrence within 2 years prior to adjuvant endocrine therapy or progression within 6 months prior to first-line endocrine therapy for metastatic $\mathrm{BCa}(22)]$ of the patients were collected from a 5-year follow-up survey for the subsequent survival analysis.

\section{RNA Extraction}

Total RNA was extracted from tissues using the TRIzol reagent (Invitrogen, Carlsbad, CA, USA). The concentration and purity of RNA were evaluated using a NanoDrop 2000 (Thermo Fisher Scientific, Waltham, MA, USA). Single stranded cDNA was synthesized from $2 \mu \mathrm{g}$ RNA using a reverse transcription reagent kit (Invitrogen) according to the manufacturer's instructions.

\section{Quantitative Real-Time PCR (qRT-PCR)}

The mRNA expression of TLR2 in tissue samples was assessed by qRT-PCR using a SYBR Green PCR kit (TaKaRa, Dalian, China) on a 7,500 Real-Time PCR System (Applied Biosystems, USA). GAPDH was used as the internal control gene, and the relative expression of TLR2 was calculated using the $2^{-\Delta \Delta \mathrm{Ct}}$ method.

\section{Statistical Analysis}

Data were expressed as the mean \pm SD and analyzed using SPSS 18.0 software (SPSS Inc., Chicago, IL, USA) and GraphPad Prism 5.0 software (GraphPad Software, Inc., USA). A K-S test was used to test the normality of TLR2. Differences between groups were analyzed using the Student's $t$-test, one-way ANOVA, or the Chisquare test. Kaplan-Meier (KM) survival curves were generated to determine the prognostic value of TLR2. A receiver operating characteristic curve (ROC) was plotted to assess the value of TLR2 for predicting the occurrence of endocrine resistance. A proportional hazards model (Cox) was used for multi-factor prognostic analysis. A $P<0.05$ was considered to indicate statistical significance. 
A

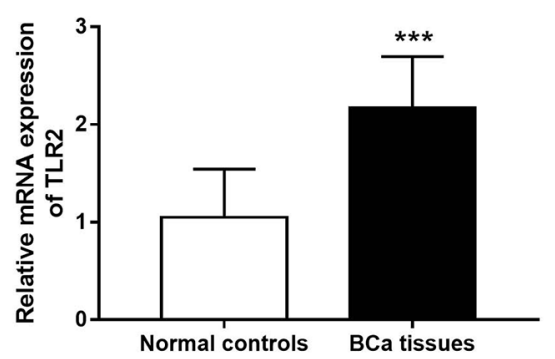

B

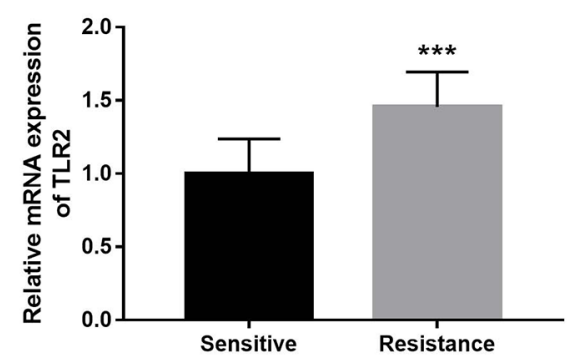

FIGURE 1 | Expression of TLR2 in BCa patients. (A) The mRNA expression of TLR2 in BCa tissues was increased compared with the normal controls. (B) The relative TLR2 expression was elevated in the BCa patients with resistance of endocrine therapy. ${ }^{\star \star \star} P<0.001$.

\section{RESULTS}

\section{Expression of TLR2 in Patients With BCa}

The results of qRT-PCR indicated that TLR2 mRNA expression was significantly higher in $\mathrm{BCa}$ tissues than in normal tissues $(P<0.001$, Figure 1A). In the 5-year follow-up, there were 24 patients with resistance to endocrine therapy, accounting for $29.3 \%$ of the $82 \mathrm{BCa}$ patients who received endocrine therapy. TLR2 mRNA expression was significantly higher in the resistant group than in the sensitive group $(P<0.001$, Figure 1B).

\section{Association of TLR2 With Clinicopathological Data of BCa Patients}

Considering that TLR2 is dysregulated in BCa tissues, this study analyzed the relationship between TLR2 expression and the clinicopathological features of BCa patients. The distribution of TLR2 was a normal distribution $(p=0.087)$. The patients were therefore divided into TLR2- low and -high expression groups according to mean TLR2 expression values. As shown in Table 1, the mRNA expression levels of TLR2 were associated with tumor size, subtypes, and TNM stage (all $P<0.05$ ). TLR2 expression was associated with HER2 status $(P=0.025)$. There was no statistically significant association between TLR2 and patient age, ER status, and PR status (all $P>0.05$ ).

\section{Prognostic Value of TLR2 in Patients With $\mathrm{BCa}$}

The value of TLR2 for predicting the prognosis of $\mathrm{BCa}$ was evaluated in the present study. In the entire BCa patient cohort, KM survival curves showed that high TLR2 expression was associated with poor overall survival compared with that of patients with low TLR2 levels (log-rank $P=0.004$, Figure 2A). In BCa patients who underwent surgery, high TLR2 mRNA levels were also associated with a shorter survival time (log-rank $P=0.037$, Figure 2B), and a similar pattern was observed in patients receiving endocrine therapy, in which high TLR2 mRNA expression was associated with poor overall survival compared with that of patients with low TLR2 expression (log-rank $P$ $=0.014$, Figure 2C) While in the entire BCa patient cohort, KM survival curves showed that high TLR2 expression was also
TABLE 1 | Relationship between TLR2 expression and clinicopathological features of BCa patients.

\begin{tabular}{|c|c|c|c|c|}
\hline \multirow[t]{2}{*}{ Features } & \multirow{2}{*}{$\begin{array}{l}\text { Total No. } \\
n=150\end{array}$} & \multicolumn{2}{|c|}{ TLR2 expression } & \multirow[t]{2}{*}{$\boldsymbol{P}$-values } \\
\hline & & Low $(n=72)$ & High $(n=78)$ & \\
\hline Age (years) & & & & 0.574 \\
\hline$\leq 50$ & 59 & 30 & 29 & \\
\hline$>50$ & 91 & 42 & 49 & \\
\hline Tumor size $(\mathrm{cm})$ & & & & $0.036^{\star}$ \\
\hline$\leq 2$ & 70 & 40 & 30 & \\
\hline$>2$ & 80 & 32 & 48 & \\
\hline ER status & & & & 0.392 \\
\hline Negative & 51 & 22 & 29 & \\
\hline Positive & 99 & 50 & 49 & \\
\hline PR status & & & & 0.647 \\
\hline Negative & 57 & 26 & 31 & \\
\hline Positive & 93 & 46 & 47 & \\
\hline HER2 status & & & & $0.025^{\star}$ \\
\hline Negative & 108 & 58 & 50 & \\
\hline Positive & 42 & 14 & 28 & \\
\hline Subtypes & & & & $0.001^{*}$ \\
\hline Luminal A & 68 & 44 & 24 & \\
\hline Luminal B & 42 & 12 & 30 & \\
\hline HER2+ & 18 & 6 & 12 & \\
\hline Triple-negative & 22 & 10 & 12 & \\
\hline TNM stage & & & & $0.030^{*}$ \\
\hline$|-I|$ & 95 & 52 & 43 & \\
\hline III-IV & 55 & 20 & 35 & \\
\hline
\end{tabular}

$E R$, estrogen receptor; $P R$, progesterone receptor; HER2, human epidermal growth factor receptor 2 ; ${ }^{*} P<0.05$.

associated with poor disease free survival compared with that of patients with low TLR2 levels (log-rank $P=0.001$, Figure 2D). Moreover, in a Cox model adjusted for method of therapy, TNM stage, level of Her2 and subtypes, patients with high level TLR2 had an elevated hazard ratio, and it was statistically significant (HR 1.87, 95\% CI: 1.02-3.40, $p=0.042$; Table 2). So, the TLR2 level was an independent role to be associated with BCa prognostic. 
A

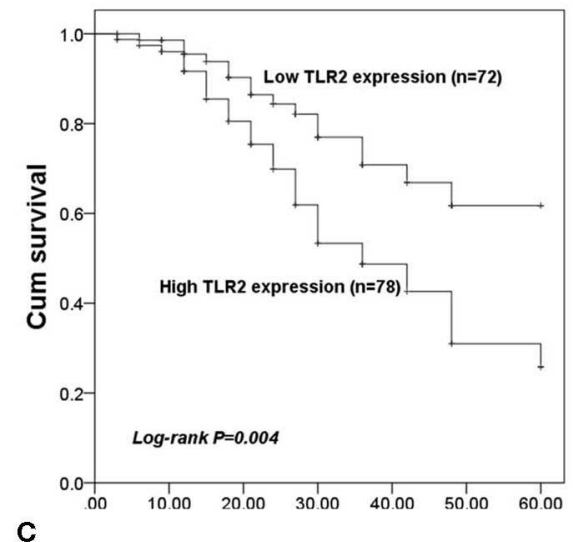

C

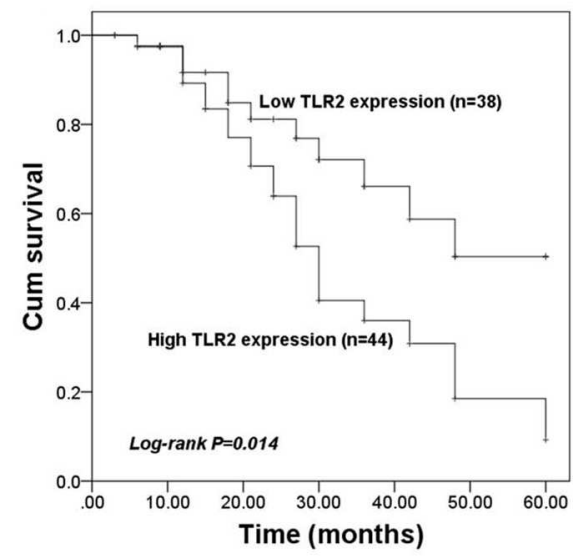

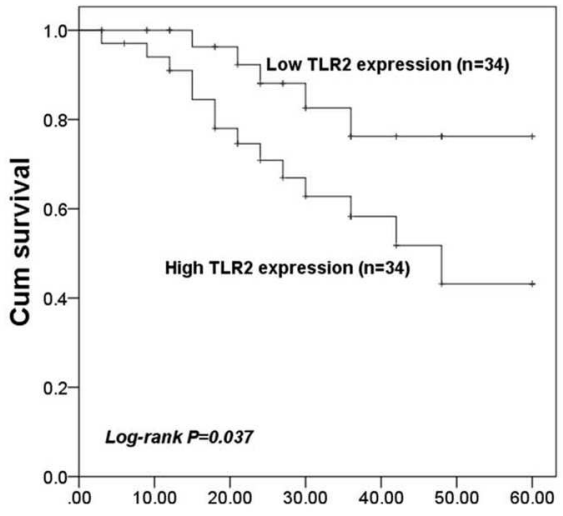

D

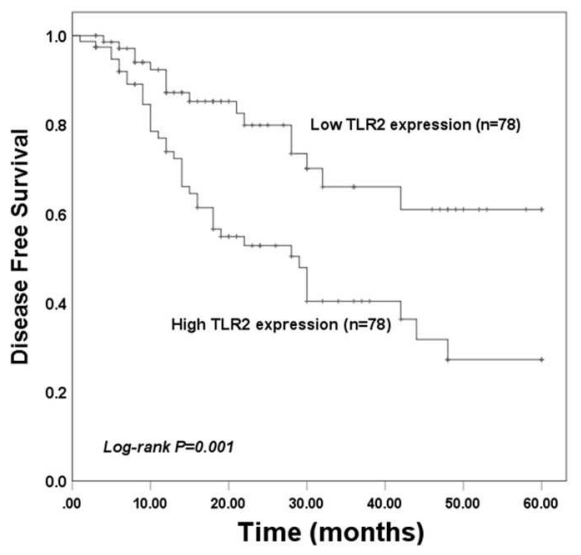

FIGURE 2 | Kaplan-Meier (KM) overall survival curves constructed with the expression of TLR2. (A) The KM curves for the total study population. (B) The KM curves for patients received surgery. (C) The KM curves for patients underwent endocrine therapy. (D) The KM disease free survival curves constructed with the expression of TLR2.

TABLE 2 | Results from a multivariate Cox model for testing the impact of TRL2 on 5-year survival; Method of therapy, TNM stage, level of Her2 and subtypes are adjustment factors.

\begin{tabular}{lccccc}
\hline Contributing factors & N & $\begin{array}{c}\text { Univariate } \\
\text { HR }\end{array}$ & HR & $\mathbf{9 5 \%} \mathbf{C l}$ & p-value \\
\hline High level of Her2 & 42 & 3.103 & 6.412 & $1.456-28.236$ & 0.014 \\
High level of TLR2 & 78 & 2.3 & 1.865 & $1.022-3.406$ & 0.042 \\
Surgery therapy & 34 & 1.809 & 1.574 & $0.899-2.757$ & 0.112 \\
TNM stage III-IV & 55 & 3.181 & 2.168 & $1.084-4.338$ & 0.029 \\
Luminal A & 68 & & & & 0.039 \\
Luminal B & 42 & 1.245 & 2.903 & $1.255-6.713$ & 0.013 \\
HER2+ & 18 & 1.31 & 4.95 & $1.098-22.222$ & 0.037 \\
Triple-negative & 22 & 4.184 & 5.495 & $1.009-30.303$ & 0.049 \\
\hline
\end{tabular}

95\% Cl, 95\% confidence interval; HR, harzard ratio.

\section{The Value of TLR2 for Predicting Endocrine Therapy Resistance}

Given the deregulated expression pattern of TLR2 and its association with poor prognosis in patients receiving endocrine therapy, this study used a ROC curve to determine the value of TLR2 for predicting the development of resistance to endocrine therapy which was the cut-off value. As shown Figure 3, the area under the curve (AUC) was 0.910, indicating a high accuracy of TLR2 for distinguishing resistant from sensitive BCa cases. At an optimal cutoff value of 1.228 , the sensitivity was $79.2 \%$ and the specificity was $86.2 \%$.

\section{Value of TLR2 for Predicting Endocrine Therapy Resistance in Different Subtypes of $\mathrm{BCa}$}

The present results suggest an association between TLR2 and HER2 and demonstrate the value of TLR2 for predicting endocrine therapy resistance. Considering HER2 status among BCa subtypes, we further investigated the clinical role of TLR2 in predicting endocrine therapy resistance by dividing $\mathrm{BCa}$ patients into luminal A $(n=45)$, HER2-negative luminal B $(n=21)$, and HER-positive luminal B cases $(n=16)$. The resistance rates among $\mathrm{BCa}$ patients are listed in Table 3. HER-positive luminal $\mathrm{B}$ patients had higher rates of resistance than luminal 
A and HER2-negative luminal B patients $(P<0.01)$, which was consistent with previous studies reporting the inhibitory effects of HER2 on endocrine therapy sensitivity (23). As shown in Figure 4A, TLR2 mRNA expression was significantly higher in HER2-positive luminal B cases than in luminal A and HER2negatie luminal B cases $(P<0.05)$. Furthermore, ROC curves based on TLR2 expression indicated that TLR2 could predict the resistance to endocrine therapy in the three subtypes; the AUC for the HER2-positive luminal B cases was relatively high among the three subtypes (AUC $=0.983$, Figures 4B-D).

\section{DISCUSSION}

$\mathrm{BCa}$ is the most common malignancy among women worldwide. This study aimed to provide a novel biomarker for predicting the prognosis of $\mathrm{BCa}$ and the resistance to endocrine therapy. The results of the present study indicated that TLR2 mRNA expression was higher in $\mathrm{BCa}$ tissues than in normal tissues and associated with tumor size, HER2 status, tumor subtype, and TNM stage. BCa patients with high TLR2 expression had a shorter overall survival than those with low TLR2 expression. In addition, TLR2 was significantly upregulated in patients showing

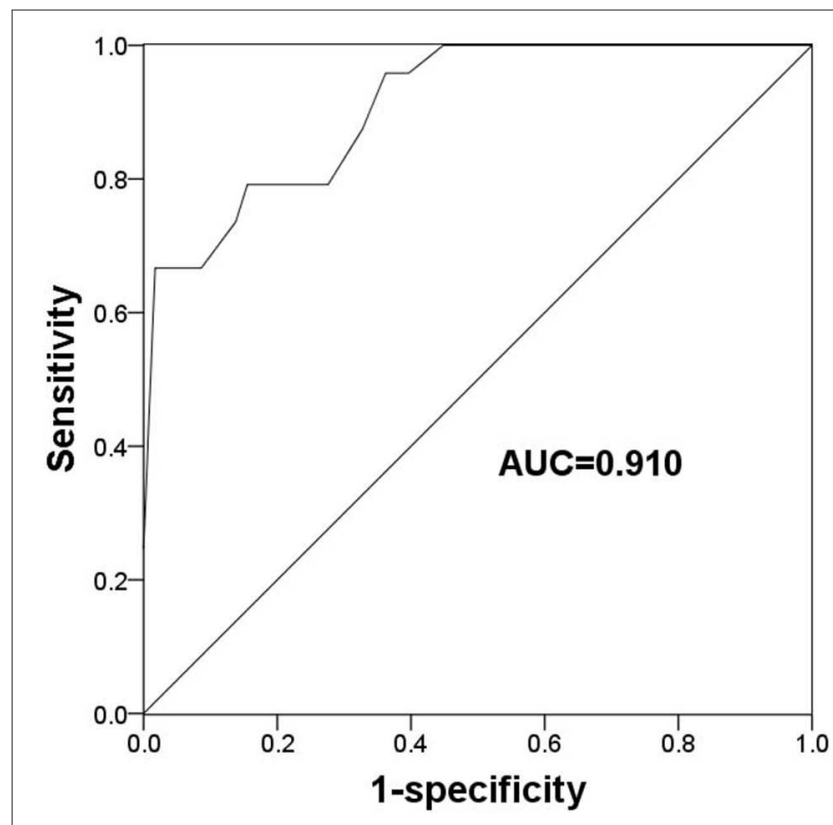

FIGURE 3 | A ROC curve to evaluate the predictive value of TLR2 for the occurrence of endocrine resistance. AUC, area under the curve. resistance to endocrine therapy, supporting its value as an indicator to predict the development of endocrine resistance in BCa patients. TLR2 expression was higher in the HER2-positive luminal $\mathrm{B} \mathrm{BCa}$ subtype than in the other hormone receptorpositive $\mathrm{BCa}$ cases, and its predictive value for the resistance to endocrine therapy was relatively high in the HER2-positive luminal B subtype.

The incidence and mortality rates of $\mathrm{BCa}$ are increasing worldwide (2). Despite advances in BCa treatment methods, the prognosis of patients with this malignancy remains unsatisfactory. Various molecules involved in the initiation and progression of BCa show aberrant expression patterns (24). Qiu et al. found that Cullin 7 is upregulated in $\mathrm{BCa}$ tissues and serves as a candidate prognostic biomarker and oncogene (25). Zhang et al. investigated the expression and clinical significance of CD81 in patients with $\mathrm{BCa}$, and provided evidence for CD81 upregulation as a prognostic biomarker and an oncogene that promotes $\mathrm{BCa}$ cell migration and proliferation (26). Dai et al. showed that CST1 is upregulated in BCa tissues, and increased CST1 expression predicts a poor prognosis and facilitates tumor progression (27). These studies prompted us to identify novel molecules showing abnormal expression that may serve as biomarkers to improve the prognosis and treatment of $\mathrm{BCa}$.

TLRs play pivotal roles in the activation of immune responses. Studies indicate that TLRs are critical mediators of chronic inflammation in the tumor microenvironment that promote the survival of tumor cells (28). TLR2 is one the most extensively investigated members of the TLR family. West et al. reported that elevated TLR2 level is associated with key genes involved in gastric cancer prognosis and identified TLR2 as a potential therapeutic target in this malignancy (29). Another study in gastric cancer provided additional evidence supporting the role of TLR2 in the inflammatory microenvironment and in promoting tumor progression (30). In glioma, TLR2 facilitates tumor cell immune evasion (31). TLR2 polymorphisms are correlated with the risk of $\mathrm{BCa}$ (10) and contribute to $\mathrm{BCa}$ cell invasion (32). However, the clinical significance of TLR2 in $\mathrm{BCa}$ remains elusive. Analysis of the survival of BCa patients indicated that patients with high TLR2 expression had a poorer prognosis than those with low TLR2 expression. These results indicate that TLR2 upregulation in $\mathrm{BCa}$ patients might predict a poor prognosis.

The development of resistance to endocrine therapy, especially in HER2-positive luminal B BCa cases, has attracted increasing attention (33). Dormant/quiescent/slow-cycle cancer stem cells (CSCs) are key factors associated with tumor heterogeneity and are responsible for tumor resistance, migration, and metastatic dormancy, which is the ability of CSCs

TABLE 3 | Endocrine therapy resistance in different BCa subtypes.

\begin{tabular}{lccccc}
\hline Indicators & Luminal A & HER2-negative luminal B & HER2-positive luminal B & $\boldsymbol{P}_{\mathbf{1}}$ & $\boldsymbol{P}_{\mathbf{2}}$ \\
\hline Total number & 45 & 21 & 16 & 0.724 & 0.002 \\
Resistance number & 9 & 5 & 10 & 0.018 \\
Resistance rate (\%) & $20.00 \%$ & $23.80 \%$ & $62.50 \%$ & & \\
\hline
\end{tabular}

HER2, human epidermal growth factor receptor 2; P1, Luminal A vs. HER2-negative luminal B; P2, Luminal A vs. HER2-positive luminal B; P3, HER2-negative luminal B vs. HER2-positive luminal $B$. 

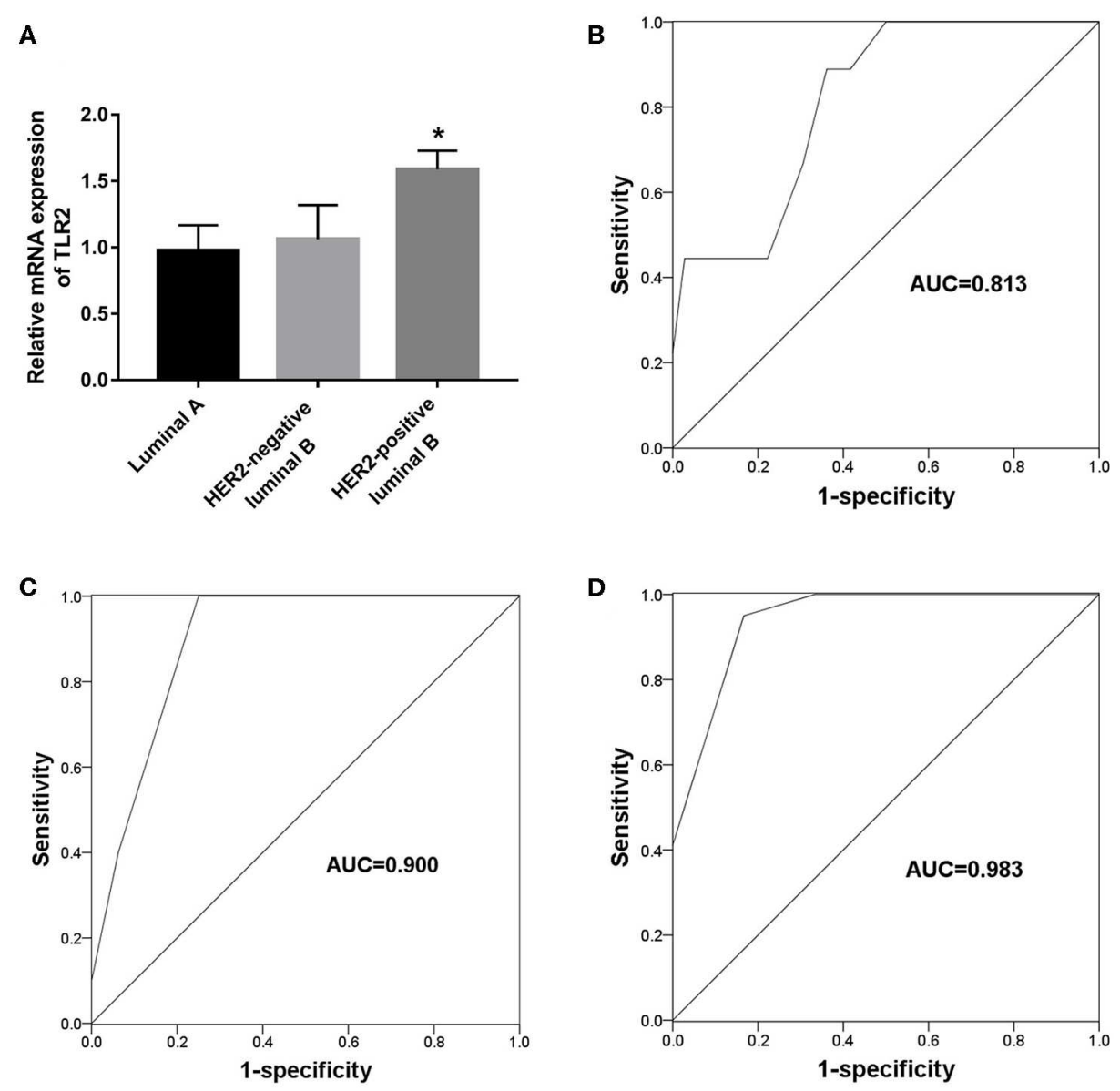

FIGURE 4 | Predictive value of TLR2 for endocrine resistance in different subtypes of BCa patients. (A) Expression of TLR2 in different subtypes of BCa patients. (B) A ROC curve to evaluate the predictive value of TLR2 for endocrine resistance in luminal $A$ subtype. (C) A ROC curve to evaluate the predictive value of TLR2 for endocrine resistance in HER-negative luminal B subtype. (D) A ROC curve to evaluate the predictive value of TLR2 for endocrine resistance in HER-positive luminal B subtype. AUC, area under the curve. ${ }^{*} p<0.05$ vs Luminal A and HER2-negative luminal B.

to survive in target organs and lead to metastasis within 20 years of diagnosis. CSCs interact with the tumor microenvironment to self-renew, resist radiation and chemotherapy, and produce distant metastases $(34,35)$. BHLHE41 (basic helix-loop-helix family, member e41) and NR2F1 (nuclear receptor subfamily 2 group F member 1) promote the growth of ER positive MCF7 cells in vivo. Analysis of patient data suggests that ER-positive diffuse tumor cells with dormancy characteristics are more likely to undergo prolonged dormancy before resuming metastatic growth. $(36,37)$.

The crosstalk between ER and HER2 may contribute to the resistance to endocrine therapy, as indicated by the enhanced HER2 activity in BCa cases treated with ER inhibitors (5). Identifying novel strategies to improve the therapeutic efficacy of endocrine therapy is critical. Keegan et al. reported that inhibiting PI3K activity may be a strategy to overcome endocrine resistance in $\mathrm{BCa}$ (38). Charmsaz et al. demonstrated that the upregulation of $\mathrm{S} 100 \beta$ in $\mathrm{BCa}$ patients is involved in endocrine resistance and serves as a promising biomarker for the response to endocrine therapy (39). Lee et al. provided evidence supporting the role of MTA1 (metastasis associated
1) as a key regulator of endocrine therapy resistance by modulating autophagy (40). In this study, TLR2 was upregulated in $\mathrm{BCa}$ patients with endocrine resistance, and its expression was effective for distinguishing resistant from sensitive cases, indicating the predictive potential of TLR2 for endocrine therapy resistance. In addition, we found an association between TLR2 and HER2. These data combined with the important role of HER2 in endocrine resistance led us to speculate that TLR2 might be involved in the development of endocrine resistance through the HER2 signaling pathway. However, this hypothesis needs to be analyzed and confirmed in further studies.

Taken together, the present data indicate that elevated TLR2 expression is a candidate prognostic biomarker in $\mathrm{BCa}$ and may predict the development of endocrine resistance. We identified a relationship between TLR2 and HER2, and showed that the value of TLR2 for predicting the response to endocrine therapy was particularly high in the HER-positive luminal B subtype, indicating that TLR2 may be involved in the regulation of endocrine therapy sensitivity through the HER signaling pathway. 
A greater number of samples and validation at different levels may be necessary to verify our results, as this study was limited by a small sample size. In addition, TLR2-related pathways need to be examined in different tissues and cells, and the possible relationship of TLR2 with dormancy in BCa should be investigated in the future.

\section{DATA AVAILABILITY STATEMENT}

The raw data supporting the conclusions of this article will be made available by the authors, without undue reservation, to any qualified researcher.

\section{REFERENCES}

1. Merino Bonilla JA, Torres Tabanera M, Ros Mendoza LH. Breast cancer in the 21st century: from early detection to new therapies. Radiologia. (2017) 59:368-79. doi: 10.1016/j.rxeng.2017.08.001

2. Bray F, Ferlay J, Soerjomataram I, Siegel RL, Torre LA, Jemal A. Global cancer statistics 2018: GLOBOCAN estimates of incidence and mortality worldwide for 36 cancers in 185 countries. CA Cancer J Clin. (2018) 68:394-424. doi: $10.3322 /$ caac. 21492

3. Harbeck N, Gnant M. Breast cancer. Lancet. (2017) 389:1134-50. doi: 10.1016/S0140-6736(16)31891-8

4. Prat A, Baselga J. The role of hormonal therapy in the management of hormonal-receptor-positive breast cancer with co-expression of HER2. Nat Clin Pract Oncol. (2008) 5:531-42. doi: 10.1038/ncponc1179

5. Arpino G, Wiechmann L, Osborne CK, Schiff R. Crosstalk between the estrogen receptor and the HER tyrosine kinase receptor family: molecular mechanism and clinical implications for endocrine therapy resistance. Endocr Rev. (2008) 29:217-33. doi: 10.1210/er.2006-0045

6. De Nardo D. Toll-like receptors: activation, signalling and transcriptional modulation. Cytokine. (2015) 74:181-9. doi: 10.1016/j.cyto.2015.02.025

7. Chen JQ, Szodoray P, Zeher M. Toll-like receptor pathways in autoimmune diseases. Clin Rev Allergy Immunol. (2016) 50:1-17. doi: 10.1007/s12016-015-8473-Z

8. Chung YH, Kim D. Enhanced TLR4 expression on colon cancer cells after chemotherapy promotes cell survival and epithelial-mesenchymal transition through phosphorylation of GSK3beta. Anticancer Res. (2016) 36:3 383-94.

9. Gambara G, Desideri M, Stoppacciaro A, Padula F, De Cesaris P, Starace D, et al. TLR3 engagement induces IRF-3-dependent apoptosis in androgensensitive prostate cancer cells and inhibits tumour growth in vivo. J Cell Mol Med. (2015) 19:327-39. doi: 10.1111/jcmm.12379

10. Zhu L, Yuan H, Jiang T, Wang R, Ma H, Zhang S. Association of TLR2 and TLR4 polymorphisms with risk of cancer: a meta-analysis. PLoS ONE. (2013) 8:e82858. doi: 10.1371/journal.pone.0082858

11. Basith S, Manavalan B, Yoo TH, Kim SG, Choi S. Roles of toll-like receptors in cancer: a double-edged sword for defense and offense. Arch Pharmacal Res. (2012) 35:1297-316. doi: 10.1007/s12272-012-0802-7

12. Takeda K, Akira S. Toll-like receptors. Curr Protoc Immunol. (2015) 109:1420. doi: 10.1002/0471142735.im1412s109

13. Paarnio K, Tuomisto A, Vayrynen SA, Vayrynen JP, Klintrup K, Ohtonen P, et al. Serum TLR2 and TLR4 levels in colorectal cancer and their association with systemic inflammatory markers, tumor characteristics, and disease outcome. APMIS Acta Pathol Microbiol Immunol Scand. (2019) 127:561-9. doi: 10.1111/apm.12971

14. Wang X, Yu X, Wang Q, Lu Y, Chen H. Expression and clinical significance of SATB1 and TLR4 in breast cancer. Oncol Lett. (2017) 14:3611-5. doi: 10.3892/ol.2017.6571

15. Chow A, Zhou W, Liu L, Fong MY, Champer J, Van Haute D, et al. Macrophage immunomodulation by breast cancer-derived exosomes requires Toll-like receptor 2-mediated activation of NF-кB. Sci Rep. (2014) 4:5750. doi: $10.1038 /$ srep 05750

\section{ETHICS STATEMENT}

The studies involving human participants were reviewed and approved by the experimental procedures were approved by the Ethics Committee of shaanxi provincial Cancer Hospital affiliated. The patients/participants provided their written informed consent to participate in this study.

\section{AUTHOR CONTRIBUTIONS}

YW and SL responsible for collecting data and writing papers. JY and $\mathrm{YZ}$ responsible for paper design.

16. Wang S, Yao Y, Rao C, Zheng G, Chen W. 25-HC decreases the sensitivity of human gastric cancer cells to 5-fluorouracil and promotes cells invasion via the TLR2/NF-кB signaling pathway. Int J Oncol. (2019) 54:966-80. doi: 10.3892/ijo.2019.4684

17. Liu B, Yan S, Jia Y, Ma J, Wu S, Xu Y, et al. TLR2 promotes human intrahepatic cholangiocarcinoma cell migration and invasion by modulating NF- $\kappa B$ pathway-mediated inflammatory responses. FEBS J. (2016) 283:383950. doi: 10.1111/febs.13894

18. Liu YD, Ji CB, Li SB, Yan F, Gu QS, Balic JJ, et al. Toll-like receptor 2 stimulation promotes colorectal cancer cell growth via PI3K/Akt and NF-кB signaling pathways. Int Immunopharmacol. (2018) 59:375-83. doi: 10.1016/j.intimp.2018.04.033

19. Lu H, Yang Y, Gad E, Inatsuka C, Wenner CA, Disis ML, et al. TLR2 agonist PSK activates human NK cells and enhances the antitumor effect of HER2targeted monoclonal antibody therapy. Clin Cancer Res. (2011) 17:6742-53. doi: 10.1158/1078-0432.CCR-11-1142

20. Goldhirsch A, Wood WC, Coates AS, Gelber RD, Thurlimann B, Senn $\mathrm{HJ}$, et al. Strategies for subtypes-dealing with the diversity of breast cancer: highlights of the St. Gallen international expert consensus on the primary therapy of early breast cancer 2011. Ann Oncol. (2011) 22:1736-47. doi: 10.1093/annonc/mdr304

21. Singletary SE, Allred C, Ashley P, Bassett LW, Berry D, Bland KI, et al. Staging system for breast cancer: revisions for the 6th edition of the AJCC Cancer staging manual. Surg Clin N Am. (2003) 83:803-19. doi: 10.1016/S0039-6109(03)00034-3

22. Cardoso F, Costa A, Senkus E, Aapro M, Andre F, Barrios CH, et al. 3rd ESOESMO international consensus guidelines for advanced breast cancer $\left(\mathrm{ABC}_{3}\right)$. Ann Oncol. (2017) 28:16-33. doi: 10.1093/annonc/mdw544

23. Ponde N, Brandao M, El-Hachem G, Werbrouck E, Piccart M. Treatment of advanced HER2-positive breast cancer: 2018 and beyond. Cancer Treat Rev. (2018) 67:10-20. doi: 10.1016/j.ctrv.2018.04.016

24. Kimbung S, Loman N, Hedenfalk I. Clinical and molecular complexity of breast cancer metastases. Semi Cancer Biol. (2015) 35:85-95. doi: 10.1016/j.semcancer.2015.08.009

25. Qiu N, He Y, Zhang S, Hu X, Chen M, Li H. Cullin 7 is a predictor of poor prognosis in breast cancer patients and is involved in the proliferation and invasion of breast cancer cells by regulating the cell cycle and microtubule stability. Oncol Rep. (2018) 39:603-10. doi: 10.3892/or.2017.6106

26. Zhang N, Zuo L, Zheng H, Li G, Hu X. Increased expression of CD81 in breast cancer tissue is associated with reduced patient prognosis and increased cell migration and proliferation in MDA-MB-231 and MDA-MB435S human breast cancer cell lines in vitro. Med Sci Monit. (2018) 24:573947. doi: 10.12659/MSM.911612

27. Dai DN, Li Y, Chen B, Du Y, Li SB, Lu SX, et al. Elevated expression of CST1 promotes breast cancer progression and predicts a poor prognosis. $\mathrm{J} \mathrm{Mol} \mathrm{Med}$. (2017) 95:873-86. doi: 10.1007/s00109-017-1537-1

28. Bhatelia K, Singh K, Singh R. TLRs: linking inflammation and breast cancer. Cell Signal. (2014) 26:2350-7. doi: 10.1016/j.cellsig.2014.07.035

29. West AC, Tang K, Tye H, Yu L, Deng N, Najdovska M, et al. Identification of a TLR2-regulated gene signature associated with tumor cell growth in gastric cancer. Oncogene. (2017) 36:5134-44. doi: 10.1038/onc.2017.121 
30. Echizen K, Hirose O, Maeda Y, Oshima M. Inflammation in gastric cancer: interplay of the COX-2/prostaglandin E2 and Toll-like receptor/MyD88 pathways. Cancer Sci. (2016) 107:391-7. doi: 10.1111/cas.12901

31. Qian J, Luo F, Yang J, Liu J, Liu R, Wang L, et al. TLR2 promotes glioma immune evasion by downregulating MHC class II molecules in microglia. Cancer Immunol Res. (2018) 6:1220-33. doi: 10.1158/2326-6066.CIR-18-0020

32. Xie W, Wang Y, Huang Y, Yang H, Wang J, Hu Z. Toll-like receptor 2 mediates invasion via activating NF- $\mathrm{B}$ in MDA-MB-231 breast cancer cells. Biochem Biophys Res Commun. (2009) 379:1027-32. doi: 10.1016/j.bbrc.2009. 01.009

33. AlFakeeh A, Brezden-Masley C. Overcoming endocrine resistance in hormone receptor-positive breast cancer. Curr Oncol. (2018) 25(Suppl. 1):S18-27. doi: 10.3747/co.25.3752

34. De Angelis ML, Francescangeli F, Zeuner A. Breast cancer stem cells as drivers of tumor chemoresistance, dormancy and relapse: new challenges and therapeutic opportunities. Cancers. (2019) 11:1569. doi: $10.3390 /$ cancers 11101569

35. Kim RS, Avivar-Valderas A, Estrada Y, Bragado P, Sosa MS, AguirreGhiso JA, et al. Dormancy signatures and metastasis in estrogen receptor positive and negative breast cancer. PLOS ONE. (2012) 7:e35569. doi: 10.1371/journal.pone.0035569

36. Adam AP, George A, Schewe D, Bragado P, Iglesias BV, Ranganathan AC, et al. Computational identification of a p38SAPK-regulated transcription factor network required for tumor cell quiescence. Cancer Res. (2009) 69:5664-72. doi: 10.1158/0008-5472.CAN-08-3820
37. Almog N, Ma L, Raychowdhury R, Schwager C, Erber R, Short $\mathrm{S}$, et al. Transcriptional switch of dormant tumors to fastgrowing angiogenic phenotype. Cancer Res. (2009) 69:836-44. doi: 10.1158/0008-5472.CAN-08-2590

38. Keegan NM, Gleeson JP, Hennessy BT, Morris PG. PI3K inhibition to overcome endocrine resistance in breast cancer. Expert Opin Investig Drugs. (2018) 27:1-15. doi: 10.1080/13543784.2018.1417384

39. Charmsaz S, Hughes E, Bane FT, Tibbitts P, McIlroy M, Byrne C, et al. S100beta as a serum marker in endocrine resistant breast cancer. BMC Med. (2017) 15:79. doi: 10.1186/s12916-017-0836-2

40. Lee MH, Koh D, Na H, Ka NL, Kim S, Kim HJ, et al. MTA1 is a novel regulator of autophagy that induces tamoxifen resistance in breast cancer cells. Autophagy. (2018) 14:812-24. doi: 10.1080/15548627.2017.1388476

Conflict of Interest: The authors declare that the research was conducted in the absence of any commercial or financial relationships that could be construed as a potential conflict of interest.

Copyright (C) 2020 Wang, Liu, Zhang and Yang. This is an open-access article distributed under the terms of the Creative Commons Attribution License (CC BY). The use, distribution or reproduction in other forums is permitted, provided the original author(s) and the copyright owner(s) are credited and that the original publication in this journal is cited, in accordance with accepted academic practice. No use, distribution or reproduction is permitted which does not comply with these terms. 